\title{
Kelvin-Helmholtz instability in magnetohydrodynamic flows
}

\author{
A. H. Khater ${ }^{1,2}$, D. K. Callebaut ${ }^{2}$, A. R. Seadawy and A. Hady ${ }^{3}$ \\ ${ }^{1}$ Department of Mathematics, Faculty of Science, Beni-Suef University , Beni-Suef, Egypt \\ email: khater_ah@yahoo.com \& aly742001@yahoo.com \\ ${ }^{2}$ Department Natuurkunde, CGB, University of Antwerp, B-2020 Antwerp, Belgium \\ email: dirk.callebaut@ua.ac.be \\ ${ }^{3}$ Department of Astronomy, Faculty of Science, Cairo University, Giza, Egypt
}

\begin{abstract}
The Rayleigh-Taylor instability (RTI) of a continuously stratified fluid has implications on the stability of solar and planetary interiors. A nonlinear stage of the two-dimensional RTI is studied by including various effects. By using the multiple scale method, we derived a nonlinear Schrödinger equation (NLSE) in $2+1$ dimensions. We show the general soliton solutions of the NLSE and this allows to discuss their stability.
\end{abstract}

Keywords. MHD; Rayleigh-Taylor instability; solar wind; planetary interiors

\section{Introduction}

The problem of RTI deals with a heavy fluid supported by a light fluid. It has implications on the stability of solar, planetary and stellar atmospheres, astrophysical, geophysical, controlled fusion, and industrial processes such as supernova explosions, and controlled thermonuclear fusion experiments (CTFs) (Chandrasekhar, 1961; Dávalos-orozco, 1993, 1996). Zelazo and Melcher pointed out that a magnetic field applied tangentially to the interface between two kinds of fluids exerts a stabilizing influence on the configuration. The stability of a magnetic fluid column was experimentally demonstrated (Zelazo and Melcher, 1969).

Malik and Singh (1989) extended the above work to a system with a magnetic field and surface tension, and demonstrated the formation of bubbles by means of the Lagrangian transformations. They showed as well how the magnetic fluid and the surface tension stabilize the interface to conserve the contours. Iizuka and Wadati (1990) studied the nonlinear stage of the RTI from a viewpoint of the nonlinear wave theory. The stabilizing effect comes from the surface tension between the two fluids and/or from the magnetic field (Malik and Singh, 1989). Dávalos-orozco $(1993,1996)$ investigated the RTI of two superposed fluids under a horizontal rotation field and also under the simultaneous action of horizontal rotation and magnetic fields (See ref. Khater et al. 2001, 2003 for details and review).

\section{Problem Formulation}

We consider two-dimensional semi-infinite, incompressible fluids separated by the interface $z=0$, along the direction of streaming. The fluid of the density $\rho_{1}$ with magnetic permeability $\mu_{1}$ occupies the half-space $z<0$, and the fluid of the density $\rho_{2}$ with magnetic permeability $\mu_{2}$ is in the half-space $z>0$. The magnetic field $\mathbf{H}(1,0,0)$ acts along the direction of the flow. The system is assumed to be irrotational under the influence of a gravitational force $\mathbf{g}(0,0,-\mathrm{g})$. The basic equations governing the velocity potential $\phi$ 
$(\mathbf{v}=\nabla \phi)$ and the magnetic potential $\psi(\mathbf{H}=-\nabla \psi)$ are

$$
\nabla^{2} \phi^{(1)}=\nabla^{2} \psi^{(1)}=0, \quad-\infty<z<\eta, \quad \nabla^{2} \phi^{(2)}=\nabla^{2} \psi^{(2)}=0, \quad \eta<z<\infty,
$$

with $\left|\nabla \phi^{(1)}\right| \rightarrow 0,\left|\nabla \psi^{(1)}\right| \rightarrow 0$ as $z \rightarrow-\infty$, and $\left|\nabla \phi^{(2)}\right| \rightarrow 0,\left|\nabla \psi^{(2)}\right| \rightarrow 0$ as $z \rightarrow \infty$. The boundary conditions at the free interface $z=\eta(x, y, t)$, are given by

$$
\begin{gathered}
\frac{\partial \phi^{(j)}}{\partial z}-\frac{\partial \eta}{\partial t}=\nabla \phi^{(j)} \cdot \nabla \eta \quad j=1,2 \quad \text { and } \quad \mu H_{n}^{(1)}=H_{n}^{(2)}, \quad H_{T}^{(1)}=H_{T}^{(2)}, \quad(2.2) \\
\rho_{1} \frac{\partial \phi^{(1)}}{\partial t}-\rho_{2} \frac{\partial \phi^{(2)}}{\partial t}+\left(\rho_{1}-\rho_{2}\right) g \eta+\frac{1}{2} \rho_{1}\left(\nabla \phi^{(1)}\right)^{2}-\frac{1}{2} \rho_{2}\left(\nabla \phi^{(2)}\right)^{2}-T\left[\nabla^{2} \eta\left(1+(\nabla \eta)^{2}\right)\right. \\
\left.-(1 / 2) \nabla \eta \cdot \nabla(\nabla \eta)^{2}\right]\left(1+(\nabla \eta)^{2}\right)^{-2 / 3}=\left(\frac{\mu_{2}(\mu-1)}{8 \pi}\right)\left(\left(H^{(1)}\right)^{2}+(\mu-1)\left(H_{n}^{(1)}\right)^{2}\right),
\end{gathered}
$$

where $\mu=\frac{\mu_{1}}{\mu 2}, H_{n}$ and $H_{T}$ represent the normal and tangential components of the magnetic field. Both the magnetic fluids are assumed to be linearly magnetizable. $T$ is the coefficient of surface tension. The nonlinear stability problem posed by Eqs. (2.1) to (2.3) is examined. The method employed is that of multiple time and space scales [Nayfeh, 1981]. In order to describe nonlinear interactions of small but finite-amplitude waves, one first normalizes the various physical quantities with respect to a characteristic length and a characteristic time. We introduce the variables

$$
x_{i}=\varepsilon^{i} x, \quad y_{i}=\varepsilon^{i} y, \quad t_{i}=\varepsilon^{i} t, \quad \eta(x, y, t)=\sum_{n=1}^{3} \varepsilon^{n} \eta_{n}\left(x_{i} ; y_{i} ; t_{i}\right)+O(\varepsilon)^{4}+\ldots,
$$

and similarly for $\phi(z, x, y, t)$ and $\psi(z, x, y, t)$, where $i=0,1,2$ and $\varepsilon$ represents a small parameter characterizing the steepness ratio of the wave. For the problem under investigation, it is sufficient to take $N=3$ as far as the lowest significant order is concerned. Eqs. (2.1)-(2.3) require a priori information of the displacement $\eta(x, y, t)$ at the perturbed surface. To circumvent this problem, we used Taylor's expansion of various quantities about $z=0$. On substituting expressions (2.4) into Eqs. (2.1) and transforming boundary conditions, and on equating the coefficients of terms of like powers in $\varepsilon$, we obtained the linear and the successive nonlinear partial differential equations (PDE's) of various orders. The problem can then be solved for any order with the knowledge of the solutions of all the previous orders.

\section{Linear theory}

The progressive wave solution of the first-order system is obtained

$$
\begin{gathered}
\eta_{1}=A(x, y, t) \exp i\left(k x_{0}+l y_{0}-\omega t_{0}\right)+c . c . \\
\phi_{1}^{(1)}=\frac{-\omega}{k} i\left(A(x, y, t) \exp i\left(k x_{0}+l y_{0}-\omega t_{0}\right)-c . c .\right) \exp (k z) \text { at } z<0 \\
\phi_{1}^{(2)}=\frac{\omega}{k} i\left(A(x, y, t) \exp i\left(k x_{0}+l y_{0}-\omega t_{0}\right)-c . c .\right) \exp (-k z) \text { at } z>0 \\
\psi_{1}^{(1)}=\frac{(1-\mu)(k+l)}{(\mu+1) k \mu_{2}} i H\left(A(x, y, t) \exp i\left(k x_{0}+l y_{0}-\omega t_{0}\right)-c . c .\right) \exp (k z) \text { at } z<0 \\
\psi_{1}^{(2)}=\frac{(1-\mu)(k+l)}{(\mu+1) k \mu_{2}} i H\left(A(x, y, t) \exp i\left(k x_{0}+l y_{0}-\omega t_{0}\right)-c . c .\right) \exp (-k z) \text { at } z>0
\end{gathered}
$$


where c.c., $A, k, l$ and $\omega$ are complex conjugate, amplitude, wavenumbers and frequency of the center of the wave packet, respectively. By substitution from (3.1)-(3.5) into (2.1)(2.3), one is led to the dispersion relation

$$
D(\omega, k, l)=\omega^{2}-k-k^{3}-k l^{2}-(k+l)^{2}\left(\frac{H^{2}}{4 \pi \mu_{2}\left(\rho_{1}+\rho_{2}\right)}\right) \frac{(1-\mu)^{2}}{\mu+1},
$$

The neutral point of the instability occurs at

$$
\frac{\left(\rho_{1}+\rho_{2}\right)^{2}}{\rho_{1} \rho_{2}}\left(\frac{k+k^{3}+k l^{2}}{(k+l)^{2}}+\left(\frac{H^{2}}{4 \rho\left(\rho_{1}+\rho_{2}\right)}\right) \frac{(1-\mu)^{2}}{\mu+1}\right)=0 .
$$

\section{Second-order problem}

With substitution of the first-order solutions (3.1)-(3.5) into the second-order equations, then the uniformly valid solutions for the second-order problem furnish the solvability condition as

$$
D_{\omega} \frac{\partial A}{\partial t_{1}}+D_{k} \frac{\partial A}{\partial x_{1}}+D_{l} \frac{\partial A}{\partial y_{1}}=0
$$

where $D_{\omega}, D_{k}$ and $D_{l}$ are the partial derivatives of the dispersion relation (3.6) with respect to frequency $\omega$ and wave numbers, respectively. The amplitude $A$ of the progressive wave is a function of the faster scales $x_{1}, x_{2} ; y_{1}, y_{2} ; t_{1}, t_{2}$. Introducing the group velocity of the wave packet $V_{k}$ and $V_{l}$ are given by

$$
V_{k}=\frac{\left(l+3 k^{2}+l^{2}\right)\left(\rho_{1}+\rho_{2}\right)+\frac{H^{2}(k+l)(\mu-1)^{2}}{2 \pi \mu_{2}(\mu+1)}}{2 \omega \rho_{1}+2 \omega \rho_{2}}, \quad V_{l}=\frac{(2 k l)\left(\rho_{1}+\rho_{2}\right)+\frac{H^{2}(k+l)(\mu-1)^{2}}{2 \pi \mu_{2}(\mu+1)}}{2 \omega \rho_{1}+2 \omega \rho_{2}},
$$

and substituting into (4.1), we get

$$
\frac{\partial A}{\partial t_{1}}+V_{k} \frac{\partial A}{\partial x_{1}}+V_{l} \frac{\partial A}{\partial y_{1}}=0
$$

\section{Third-order problem}

By using the first-order and the second-order solutions, Simplifying the right-hand side of third order equations and after some straightforward reductions, the condition for the free surface elevation $\eta_{3}$ to be nonsecular is

$$
2 i\left[\frac{\partial A}{\partial t_{2}}+V_{k} \frac{\partial A}{\partial x_{2}}+V_{l} \frac{\partial A}{\partial y_{2}}\right]+R_{1} \frac{\partial^{2} A}{\partial x_{1}^{2}}+2 R_{2} \frac{\partial^{2} A}{\partial x_{1} \partial y_{1}}+R_{3} \frac{\partial^{2} A}{\partial y_{1}^{2}}=Q_{1}|A|^{2} A
$$

where

$$
\begin{gathered}
R_{1}=\Omega-\frac{\left(3 k-V_{k}^{2}\right) \rho_{1}+\left(3 k-V_{k}^{2}\right) \rho_{2}}{\omega \rho_{1}+\omega \rho_{2}}, \quad R_{2}=\Omega-\frac{\left(l-V_{l} V_{k}\right) \rho_{1}+\left(l-V_{l} V_{k}\right) \rho_{2}}{\omega \rho_{1}+\omega \rho_{2}} \\
R_{3}=\Omega-\frac{\left(k-V_{l}^{2}\right) \rho_{1}+\left(k-V_{l}^{2}\right) \rho_{2}}{\omega \rho_{1}+\omega \rho_{2}} \\
Q_{1}=\frac{4 \rho_{1} \omega^{2}-4 \rho_{2} \omega^{2}-\frac{(1-\mu)^{2}}{(1+\mu)} \frac{\left(k^{2}+l^{2}\right) H^{2}}{\rho}}{\left(-1+2 k^{2}+2 l^{2}\right)\left(\omega \rho_{1}+\omega \rho_{2}\right)}-\frac{\left(4 k^{2}+4 l^{2}-k^{4}-l^{4}\right)\left(\rho_{1}+\rho_{2}\right)}{\omega \rho_{1}+\omega \rho_{2}}
\end{gathered}
$$


where $\Omega=\frac{-H^{2}(\mu-1)^{2}}{4 \pi \mu_{2}(\mu+1)\left(\omega \rho_{1}+\omega \rho_{2}\right)}$. Introducing the transformations

$$
\xi_{1}=\frac{x_{1}}{R_{1}^{1 / 2}}, \quad \eta_{1}=\left(\frac{R_{2}^{2}}{R_{1}}-R_{3}\right)^{-1 / 2}\left(\frac{R_{2}}{R_{1}} x_{1}-y_{1}\right), \quad \tau=t_{2},
$$

Under such a transformations, (5.2) reduced to an elliptic two-dimensional NLSE

$$
2 i \frac{\partial A}{\partial \tau}+\frac{\partial^{2} A}{\partial \xi_{1}^{2}}-\frac{\partial^{2} A}{\partial \eta_{1}^{2}}=Q|A|^{2} A
$$

where $Q=\frac{Q_{1}}{R_{1}}$. Eq. (5.4) has soliton solutions as (Khater et al. 2001, 2003)

$$
\begin{aligned}
A=(\sqrt{2 C / Q} & \left.\sec \left(\sqrt{C}\left(\ln \left[\exp \left(d_{1} \eta_{1}\right) \sin \left(d_{0} \tau+d_{1} \xi_{1}\right)\right]\right)+d\right)-\pi\right) \exp i\left(c_{0} \tau+c_{1} \xi_{1}+c_{2} \eta_{1}\right) \\
A= & \sqrt{\frac{C}{Q}} \sinh \left(2 \tanh ^{-1}\left(\exp \left(\sqrt{2 C}\left(d_{0} \tau+d_{1} \xi_{1}+d_{2} \eta_{1}\right)+d\right)\right)-\pi\right) \\
& \times \exp \left(i\left(c_{0} \tau+c_{1} \xi_{1}+c_{2} \eta_{1}\right)\right)
\end{aligned}
$$

where $C, d_{0}, d_{1}, d_{2}, c_{0}, c_{1}, c_{2}$ are real constants. The soliton stability of Eq. (5.3) can be obtained by deriving the functional relation $\delta L / \delta A^{*}=0$, from the Lagrangian

$$
L=\iint\left(\frac{i}{2}\left(A^{*} \frac{\partial A}{\partial \tau}-A \frac{\partial A^{*}}{\partial \tau}\right)-\frac{1}{2}\left(\left|\frac{\partial A}{\partial \xi_{1}}\right|^{2}-\left|\frac{\partial A}{\partial \eta_{1}}\right|^{2}+\frac{Q}{2}|A|^{2}\right)\right) d \xi_{1} d \eta_{1} .
$$

We have two integrals of motion as

$$
N=\iint\left(|A|^{2}\right) d \xi_{1} d \eta_{1}, \quad H=\frac{1}{2} \iint\left(\left|\frac{\partial A}{\partial \xi_{1}}\right|^{2}-\left|\frac{\partial A}{\partial \eta_{1}}\right|^{2}+\frac{Q}{2}|A|^{2}\right) d \xi_{1} d \eta_{1},
$$

where the normalization $N$ is the wave action and $H$ is the Hamiltonian.

\section{Conclusion}

The nonlinear stage of the two-dimensional RTI is studied by including various effects, even the effect of surface tension between the two fluids. By using the multiple scales method. Linearized equations for the system are derived and the dispersion relation is obtained. From the second- and third-order theories, we have derived a NLSE in $2+1$ dimensions. We find the soliton solutions of $2+1$ dimensions NLSE. This allows to discuss the stability of the soliton solutions.

\section{References}

Chandrasekhar, S., 1961, Hydrodynamic and Hydromagnetic Stability (Clarendon Press)

Dávalos-orozco, L. A., 1993, Fluid Dynamics Research, 12, 243

Dávalos-orozco, L. A., 1996, Dynamics of atmospheres and oceans, 23, 274

Dávalos-orozco, L. A., 1996, Astrophysics and space science, 243, 291

Iizuka, T. \& Wadati, M., 1990, J. Phys. Soc. Jpn., 59, 3182

Khater, A. H., Callebaut, D. K., Malfliet, W. \& Seadawy, A. R., 2001, Physica Scripta, 64, 533

Khater, A. H., Callebaut, D. K. \& Seadawy, A. R., 2003, Physica Scripta, 67, 340 